\title{
Separating the direct effects of traits on atherosclerotic cardiovascular disease from those mediated by type 2 diabetes
}

\author{
Venexia M. Walker ${ }^{1,2,3}$ (D) Marijana Vujkovic ${ }^{4}$ (D) $\cdot$ Alice R. Carter $^{1,2}$ (D) Neil M. Davies $^{1,2,5}$ (D) Miriam S. Udler ${ }^{6}$ (D) \\ Michael G. Levin ${ }^{7,8}$ (D) George Davey Smith ${ }^{1,2}$ (D) Benjamin F. Voight ${ }^{9,10,11}$ (D) Tom R. Gaunt $^{1,2}$ (D) \\ Scott M. Damrauer ${ }^{3,8,10}$
}

Received: 5 August 2021 / Accepted: 22 November 2021 / Published online: 7 February 2022

(C) The Author(s) 2022

\begin{abstract}
Aims/hypothesis Type 2 diabetes and atherosclerotic CVD share many risk factors. This study aimed to systematically assess a broad range of continuous traits to separate their direct effects on coronary and peripheral artery disease from those mediated by type 2 diabetes.

Methods Our main analysis was a two-step Mendelian randomisation for mediation to quantify the extent to which the associations observed between continuous traits and liability to atherosclerotic CVD were mediated by liability to type 2 diabetes. To support this analysis, we performed several univariate Mendelian randomisation analyses to examine the associations between our continuous traits, liability to type 2 diabetes and liability to atherosclerotic CVD.

Results Eight traits were eligible for the two-step Mendelian randomisation with liability to coronary artery disease as the outcome and we found similar direct and total effects in most cases. Exceptions included fasting insulin and hip circumference where the proportion mediated by liability to type 2 diabetes was estimated as $56 \%$ and $52 \%$, respectively. Six traits were eligible for the analysis with liability to peripheral artery disease as the outcome. Again, we found limited evidence to support mediation by liability to type 2 diabetes for all traits apart from fasting insulin (proportion mediated: $70 \%$ ).

Conclusions/interpretation Most traits were found to affect liability to atherosclerotic CVD independently of their relationship with liability to type 2 diabetes. These traits are therefore important for understanding atherosclerotic CVD risk regardless of an individual's liability to type 2 diabetes.
\end{abstract}

Keywords Atherosclerotic cardiovascular disease - Coronary artery disease - Direct effect - Genome-wide association study . Indirect effect $\cdot$ Mediation $\cdot$ Mendelian randomisation $\cdot$ Peripheral artery disease $\cdot$ Type 2 diabetes

Venexia M. Walker

venexia.walker@bristol.ac.uk

1 MRC University of Bristol Integrative Epidemiology Unit, Bristol, UK

2 Bristol Medical School: Population Health Sciences, University of Bristol, Bristol, UK

3 Department of Surgery, University of Pennsylvania Perelman School of Medicine, Philadelphia, PA, USA

4 Department of Medicine, University of Pennsylvania Perelman School of Medicine, Philadelphia, PA, USA

5 K.G. Jebsen Center for Genetic Epidemiology, Department of Public Health and Nursing, NTNU, Norwegian University of Science and Technology, Trondheim, Norway
6 Center for Genomic Medicine, Massachusetts General Hospital, Boston, MA, USA

7 Division of Cardiovascular Medicine, University of Pennsylvania Perelman School of Medicine, Philadelphia, PA, USA

8 Corporal Michael Crescenz VA Medical Center, Philadelphia, PA, USA

9 Department of Systems Pharmacology and Translational Therapeutics, University of Pennsylvania Perelman School of Medicine, Philadelphia, PA, USA

10 Department of Genetics, University of Pennsylvania Perelman School of Medicine, Philadelphia, PA, USA

11 Institute of Translational Medicine and Therapeutics, University of Pennsylvania Perelman School of Medicine, Philadelphia, PA, USA 


\section{Research in context}

\section{What is already known about this subject?}

- Type 2 diabetes, coronary artery disease and peripheral artery disease share many common risk factors

- Type 2 diabetes is also one of the strongest independent risk factors for both coronary and peripheral artery disease

\section{What is the key question?}

- $\quad$ For traits associated with atherosclerotic CVD, what proportion of their effect is mediated by liability to type 2 diabetes?

\section{What are the new findings?}

- Using two-step Mendelian randomisation for mediation, we found that most traits for atherosclerotic CVD were likely to act independently of liability to type 2 diabetes

- The effects of fasting insulin and hip circumference on liability to coronary artery disease were partially mediated, with estimates for the proportion mediated of $56 \%$ and $52 \%$, respectively

- The effects of fasting insulin on liability to peripheral artery disease also indicated partial mediation, with an estimate for the proportion mediated of $70 \%$

\section{How might this impact on clinical practice in the foreseeable future?}

- Traits that are likely to affect liability to atherosclerotic CVD independently of their relationship with liability to type 2 diabetes are important for understanding atherosclerotic CVD risk, regardless of an individual's liability to type 2 diabetes

\section{Abbreviations \\ FDR False discovery rate \\ GWAS Genome-wide association studies}

\section{Introduction}

Type 2 diabetes shares several risk factors with the atherosclerotic CVDs coronary artery disease and peripheral artery disease. These risk factors include obesity and hypertension [1-3]. In addition, type 2 diabetes is one of the strongest independent risk factors for both coronary and peripheral artery disease $[4,5]$. As a result of the shared links between type 2 diabetes and atherosclerotic CVD, it can be difficult to separate the direct effects of risk factors for atherosclerotic CVD from those mediated by type 2 diabetes. Distinguishing these effects is important because it may provide novel biological insight into the conditions individually, while also improving our understanding of their commonalities.

Mendelian randomisation uses genetic variants associated with an exposure (referred to as an 'instrument') as a proxy for that exposure [6]. This method can be used to estimate the causal effect of an exposure on an outcome free from bias due to non-genetic confounding and reverse causality if its assumptions hold [7]. Two-step Mendelian randomisation for mediation analysis is an extension to this method and incorporates the causal effect of a mediator, to estimate the direct (independent of the mediator) and indirect (via the mediator) effects of an exposure on an outcome [8, 9]. Furthermore, this approach can be applied using summary statistics from multiple genome-wide association studies (GWASs) with non-overlapping samples [10]. This removes the need for individual-level data from a single study containing information on all the risk factors, allowing broad systematic assessment of a wide range of risk factors unlikely to be captured in one place.

While Mendelian randomisation has previously been used to individually estimate the effect of several risk factors on liability to our three disease outcomes of interest [4, 11-15], and Mendelian randomisation for mediation has been conducted to investigate the mediating effect of a selected set of obesityrelated markers [16], systematic assessment of a wide range of traits using Mendelian randomisation to separate their effects on liability to atherosclerotic CVD from liability to type 2 diabetes has not yet been conducted. The aim of this study was therefore to implement a standardised univariate Mendelian randomisation framework and follow-up analyses with twostep Mendelian randomisation for mediation to interrogate the association of a broad range of continuous traits with liability to our three disease outcomes: type 2 diabetes; coronary artery disease; and peripheral artery disease. 


\section{Methods}

Study design Our study consisted of two stages (summarised in Fig. 1). First, we used univariate Mendelian randomisation to estimate the effects of 108 continuous traits (see Trait selection, below) on liability to three disease outcomes: type 2 diabetes; coronary artery disease; and peripheral artery disease. In addition, we used univariate Mendelian randomisation to estimate the effect of liability to type 2 diabetes on the 108 continuous traits. This allowed us to remove traits that had a bidirectional association with liability to type 2 diabetes as we cannot determine which phenotype should be the exposure and which the mediator for downstream analyses in this case. Based on the evidence from stage 1, we implemented stage 2: two-step Mendelian randomisation for mediation. Using this approach, we estimated the direct effect (i.e. independent of liability to type 2 diabetes) and indirect effect (i.e. mediated via liability to type 2 diabetes) of the traits on the atherosclerotic CVDs of interest.

Trait selection Traits were selected from the IEU OpenGWAS database by implementing a selection procedure to retain the largest, minimally adjusted GWAS for each continuous biological trait that had been studied in both men and women of European or mixed ancestry (electronic supplementary material [ESM] Fig. 1) [17]. Sample overlap was permitted between traits and so most of the GWASs included participants from UK Biobank [18].

Outcome phenotypes We obtained the GWASs for liability to type 2 diabetes in European ancestry from the DIAMANTE consortium [19]. The GWASs for liability to coronary artery disease and liability to peripheral artery disease were obtained from the CARDIoGRAM consortium and Million Veteran Program, respectively [20-22]. As noted above, sample overlap was permitted between traits, although GWASs were obtained from distinct samples for liability to type 2 diabetes, coronary artery disease and peripheral artery disease.

Univariate Mendelian randomisation Instruments for each trait were defined using the genome-wide significant $(p<5 \times$ $10^{-8}$ ) genetic variants from the corresponding GWAS to satisfy the first instrumental variable assumption of relevance. A description of relevance and the other the instrument assumptions required for Mendelian randomisation are given in ESM Methods 1. For the univariate Mendelian randomisation analyses, instruments were clumped using a $10 \mathrm{Mb}$ window and $R^{2}$ linkage disequilibrium (LD) threshold of 0.001 against the 1000 genomes reference panel for the European super-population, which was filtered to include only bi-allelic variants with minor allele frequencies greater than 0.01 . Instruments

a

\begin{tabular}{cccc}
\multicolumn{3}{c}{ Stage 1} & Stage 2 \\
\hline $\begin{array}{c}\text { UVMR evidence for } \\
\text { risk factor }>\text { T2D }\end{array}$ & $\begin{array}{c}\text { UVMR evidence for } \\
\text { T2D }>\text { risk factor }\end{array}$ & $\begin{array}{c}\text { UVMR evidence for } \\
\text { risk factor }>\text { ASCVD }\end{array}$ & $\begin{array}{c}\text { Two-step MR } \\
\text { for mediation }\end{array}$ \\
\hline Supportive & Supportive & Supportive & None \\
Supportive & Limited & Supportive & See b \\
Supportive & Supportive & Limited & None \\
Supportive & Limited & Limited & None
\end{tabular}

b

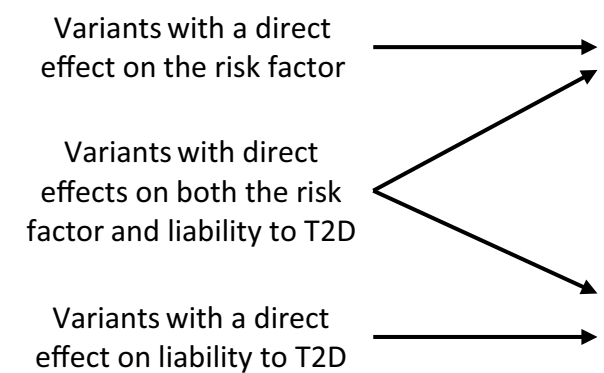

Fig. 1 Illustration of the two-stage study design and the two-step Mendelian randomisation for mediation model used for stage 2. (a) Summary of how evidence from univariate Mendelian randomisation analyses of the risk factor, liability to type 2 diabetes, and liability to atherosclerotic CVD are assessed in stage 1 . Here, estimates that met the arbitrary FDR threshold of 5\% were deemed to lend 'supportive' evidence, while all other estimates were considered to provide 'limited'
Risk factor

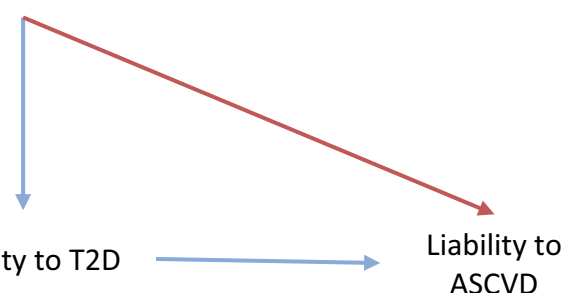

evidence. Depending on the evidence obtained in stage 1, a trait may progress to stage 2 (i.e. the two-step Mendelian randomisation for mediation). (b) The model for two-step Mendelian randomisation is shown. Red arrow represents the direct (i.e. independent of the mediator) effect and the blue arrow represents the indirect (i.e. via the mediator) effect. ASCVD, atherosclerotic CVD; T2D, type 2 diabetes; UVMR, univariate Mendelian randomisation; MR, Mendelian randomisation 
consisting of less than ten variants were removed, before harmonisation with the outcome data to represent an increase in the exposure. Mendelian randomisation was then performed using the inverse variance weighted method. Note that all estimates are presented in SD units to allow comparison between traits.

We repeated the above univariate Mendelian randomisation analyses using the simple mode, weighted median, weighted mode and MR-Egger methods as a sensitivity analysis to examine estimate consistency. We also derived heterogeneity statistics to examine the consistency of estimates across the variants included in each analysis and performed a leave-one-out analysis to determine whether certain variants were driving the observed effects. We included an MR-Egger intercept test to assess whether directional pleiotropy was likely to have affected our results [23]. Finally, to assess the no measurement error assumption for MR-Egger, we calculated the $I_{G X}^{2}$ statistic as a measure of potential attenuation bias [24]. All univariate analyses and associated sensitivity analyses were implemented using the TwoSampleMR package for $\mathrm{R}$ [25].

Two-step Mendelian randomisation for mediation When we found the following evidence: (1) evidence to support an effect of the trait on liability to type 2 diabetes; (2) limited evidence to support an effect of liability to type 2 diabetes on the trait; and (3) evidence of an effect on liability to at least one atherosclerotic cardiovascular outcome of interest, multivariable Mendelian randomisation was applied using the trait and liability to type 2 diabetes as exposures. An arbitrary false discovery rate (FDR) of 5\%, calculated according to the Benjamini and Hochberg method, was used as an indicator of supportive evidence of an association [26]. This multivariable Mendelian randomisation allowed us to estimate the effect of the trait, independent of liability to type 2 diabetes, on the liability to atherosclerotic CVD outcome of interest (Fig. 1b). This effect is often referred to as the 'direct' effect. We were also able to derive the effect of the trait, through liability to type 2 diabetes on liability to the atherosclerotic CVD outcome of interest, often referred to as the 'indirect' or 'mediated' effect. For the two-step Mendelian randomisation for mediation, we multiplied the estimate for the effect of the trait on liability to type 2 diabetes obtained from the univariate Mendelian randomisation by the direct effect of liability to type 2 diabetes on the atherosclerotic cardiovascular outcome of interest obtained from the multivariable Mendelian randomisation (where the exposure of interest and mediator were both used as exposures). CIs were derived using the sum of squares method.

Instruments for this analysis were clumped against either the trait or liability to type 2 diabetes (whichever had the smallest instrument) using a $10 \mathrm{Mb}$ window and $R^{2} \mathrm{LD}$ threshold of 0.001 against the 1000 genomes reference panel for the European super-population, which was filtered to include only bi-allelic variants with minor allele frequencies greater than 0.01 . Harmonisation was performed with variants aligned to represent an increase in the trait prior to analysis. We calculated conditional $F$ statistics to test instrument strength for each exposure in our analysis. We also calculated a modified form of Cochran's $Q$ statistic that has been developed to measure heterogeneity in causal effect estimates from multivariable Mendelian randomisation. Multivariable Mendelian randomisation estimates and these statistics were obtained using the MVMR package for R [27]. Again, all estimates are presented in SD units to allow comparison between traits. The non-collapsibility of ORs can pose a problem when using summary statistics from logistic regression for binary mediators and outcomes in multivariable Mendelian randomisation. To assess whether this is likely to have impacted our results, we repeated our analyses using a GWAS of liability to type 2 diabetes based on a linear (instead of a logistic) model (ESM Methods 2). Finally, note that alongside the standard instrument assumptions required for Mendelian randomisation (ESM Methods 1), two-step Mendelian randomisation for mediation also assumes no interaction between the exposure and the mediator.

Code availability All analyses were conducted in R version 4.0.2. The associated code is available from https:/github. $\mathrm{com} /$ venexia/T2DMediationMR.

Ethics approval This research using UK Biobank data was completed under Application Number 15825, which has been subject to ethics approval.

\section{Results}

The results of this analysis are presented in four parts: (1) the selection of traits from the IEU OpenGWAS database [17]; (2) the results of the univariate Mendelian randomisation analyses to interrogate the effect of each trait on liability to type 2 diabetes and the effect of liability to type 2 diabetes on each trait; (3) the results related to liability to coronary artery disease from both the univariate Mendelian randomisation and two-step Mendelian randomisation for mediation; and (4) results related to liability to peripheral artery disease from the univariate Mendelian randomisation and two-step Mendelian randomisation for mediation.

Trait selection We identified 108 traits from the IEU OpenGWAS database for inclusion in our analysis [17]. Details of both the trait and outcome GWASs are provided in ESM Table 1. Most of the trait GWASs were conducted in UK Biobank by the Neale lab [28]. Twelve of the selected GWASs were from other sources: adiponectin [29]; alcoholic 
drinks per week [30]; body fat [31]; BMI [32]; cigarettes per day [30]; fasting glucose [33]; fasting insulin [33]; heart rate [34]; neuroticism [35]; total cholesterol [36]; urinary sodiumpotassium ratio [37]; and waist/hip ratio [38].

\section{Causes and consequences of liability to type 2 diabetes}

Estimates from bidirectional univariate Mendelian randomisation of each trait and liability to type 2 diabetes found evidence for ten traits as causes, but not consequences, of liability to type 2 diabetes at an FDR threshold of 5\% (ESM Figs 2, 3; ESM Table 2). These traits were taken forward to the two-step Mendelian randomisation for mediation analyses for liability to atherosclerotic CVD. Sensitivity analyses using alternative Mendelian randomisation methods were consistent with the inverse variance weighted estimates (ESM Table 2). The MR-Egger intercept test found intercepts between -0.15 (body fat on liability to type 2 diabetes) and 0.07 (fasting glucose on liability to type 2 diabetes) (ESM Table 3 ). Finally, the $I_{G X}^{2}$ statistic was over 0.93 for all MR-Egger results (ESM Table 4). When taken as an estimate of the attenuation bias in these analyses, this corresponds to less than $7 \%$ relative bias towards the null.

Causes of liability to coronary artery disease Using univariate Mendelian randomisation, we found evidence for 53 of the 108 traits as causes of liability to coronary artery disease at the FDR threshold of 5\% (ESM Fig. 4; ESM Table 2). After restricting to traits thought to be causes of liability to type 2 diabetes, eight traits remained and were studied using twostep Mendelian randomisation for mediation (ESM Table 5, where variable 'stage2 cad' is true). In this analysis, we found similar direct and total effects for most traits: apolipoprotein B; aspartate aminotransferase; diastolic BP; standing height; total cholesterol; and trunk fat percentage (Fig. 2 and ESM Table 6). The exceptions were fasting insulin and hip circumference, where the effects indicated partial mediation by liability to type 2 diabetes. These effects corresponded to an estimate for the proportion of the effect mediated by liability to type 2 diabetes of $56 \%$ and $52 \%$, respectively (ESM Table 7). The conditional $F$ statistics for the multivariable Mendelian randomisation component of these analyses ranged from 9 to 87 (ESM Table 6), indicating good instrument strength. Meanwhile, the modified Cochran's $Q$ statistic exceeded the critical value for the $\chi^{2}$ distribution at the $5 \%$ level for all analyses. This indicated that the chosen SNPs predicted both the trait and liability to type 2 diabetes in the data. Taken as a whole, the analyses concerning liability to coronary artery disease suggest that the effects of the traits are likely to be independent of the effects of liability to type 2 diabetes.

Causes of liability to peripheral artery disease We found evidence for 42 traits as causes of liability to peripheral artery disease at the FDR threshold of $5 \%$ using univariate
Mendelian randomisation (ESM Fig. 5; ESM Table 2). After restricting to traits thought to be causes of liability to type 2 diabetes, six traits remained: apolipoprotein B, diastolic BP, fasting insulin, hip circumference, total cholesterol and trunk fat percentage (ESM Table 5, where variable 'stage2 pad' is true). Two-step Mendelian randomisation for mediation of these traits found similar direct and total effects in most cases (Fig. 3; ESM Table 6). Fasting insulin was again identified as an exception with effects that indicated partial mediation by liability to type 2 diabetes and an estimated proportion mediated of 70\% (ESM Table 7). The conditional $F$ statistics for the multivariable Mendelian randomisation component of these analyses again indicated good instrument strength, ranging from 9 to 86 (ESM Table 6). We also found the modified Cochran's $Q$ statistic exceeded the critical value for the $\chi^{2}$ distribution at the $5 \%$ level for all liability to peripheral artery disease analyses. Similar to the results concerning liability to coronary artery disease, these analyses suggest that the effects for most of the traits on liability to peripheral artery disease are likely to be independent of the effects of liability to type 2 diabetes.

\section{Discussion}

Using univariate Mendelian randomisation, we provide evidence for the causal effects of multiple traits on liability to our three outcomes of interest: type 2 diabetes; coronary artery disease; and peripheral artery disease, (Fig. 4). Common traits for liability to these outcomes included glycaemic traits such as glucose (type 2 diabetes, OR 3.34 [95\% CI 2.41, 4.63] [ESM Fig. 2]; coronary artery disease, OR 1.25 [95\% CI 1.11, 1.41] [ESM Fig. 4]; peripheral artery disease, OR 1.26 [95\% CI 1.10, 1.44] [ESM Fig. 5]) and anthropometric traits such as body fat percentage (type 2 diabetes, OR 2.78 [95\% CI 2.32, 3.32] [ESM Fig. 2]; coronary artery disease, OR 1.52 [95\% CI 1.33, 1.73] [ESM Fig. 4]; peripheral artery disease, OR 1.92 [95\% CI 1.68, 2.19] [ESM Fig. 5]). We also identified specific traits for each outcome. For instance, there were five traits with evidence to support an effect on liability to type 2 diabetes (whole-body fat-free mass, whole-body water mass, peak expiratory flow, lymphocyte count, IGF-1) but not liability to coronary or peripheral artery disease, as well as 12 and eight traits with specific effects on liability to coronary and peripheral artery disease, respectively. These findings confirm several known traits for each of the outcomes and may provide novel biological insight regarding some of the lesser-studied traits.

Using two-step Mendelian randomisation for mediation analysis, this study found that the effects of most of the eligible traits were likely to be independent of the effects of liability to type 2 diabetes. There are several reasons why a mediating effect may not have been identified in this analysis. First, 
Fig. 2 Two-step Mendelian randomisation for mediation estimates for the total, indirect (mediated by liability to type 2 diabetes) and direct (independent of liability to type 2 diabetes) effects of the indicated risk factors on liability to coronary artery disease
Total, OR $1.80(95 \% \mathrm{Cl} 1.60,2.02)$
Indirect, OR $0.98(95 \% \mathrm{Cl} 0.89,1.08)$
Direct, OR $1.55(95 \% \mathrm{Cl} 1.40,1.71)-$

Total, OR $1.20(95 \% \mathrm{Cl} 1.07,1.34)$
Indirect, OR $1.02(95 \% \mathrm{Cl} 0.92,1.13)-\mid$
Direct, OR $1.15(95 \% \mathrm{Cl} 1.02,1.31)-$

Total, OR $1.79(95 \% \mathrm{Cl} 1.54,2.08)$
Indirect, OR $1.03(95 \% \mathrm{Cl} 0.91,1.17)-$
Direct, OR $1.63(95 \% \mathrm{Cl} 1.41,1.87)-1$

Total, OR $1.48(95 \% \mathrm{Cl} 1.20,1.82)$
Indirect, OR $1.25(95 \% \mathrm{Cl} 0.85,1.83)$
Direct, OR $1.23(95 \% \mathrm{Cl} 1.02,1.47)-$

Total, OR $1.14(95 \% \mathrm{Cl} 1.03,1.26)$
Indirect, OR $1.07(95 \% \mathrm{Cl} 0.91,1.26)-$
Direct, OR $1.06(95 \% \mathrm{Cl} 0.96,1.18)-$

Total, OR $0.81(95 \% \mathrm{Cl} 0.77,0.86)$
Indirect, OR $0.99(95 \% \mathrm{Cl} 0.92,1.06)-$
Direct, OR $0.78(95 \% \mathrm{Cl} 0.71,0.85)-1$

Total, OR $1.52(95 \% \mathrm{Cl} 1.37,1.69)$
Indirect, OR $0.98(95 \% \mathrm{Cl} 0.88,1.09)-$
Direct, OR $1.52(95 \% \mathrm{Cl} 1.39,1.67)-1$

Total, OR $1.27(95 \% \mathrm{Cl} 1.13,1.43)$
Indirect, OR $1.11(95 \% \mathrm{Cl} 0.94,1.32)$
Direct, OR $1.25(95 \% \mathrm{Cl} 1.10,1.42)$

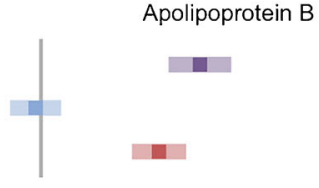

Aspartate aminotransferase

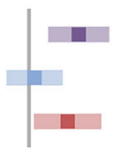

Diastolic blood pressure

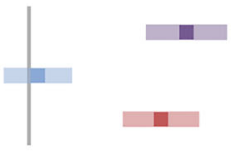

Fasting insulin

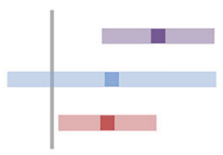

Hip circumference

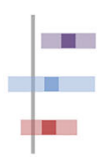

Standing height

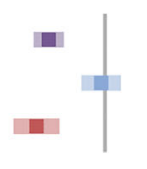

Total cholesterol

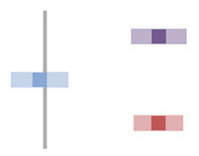

Trunk fat percentage

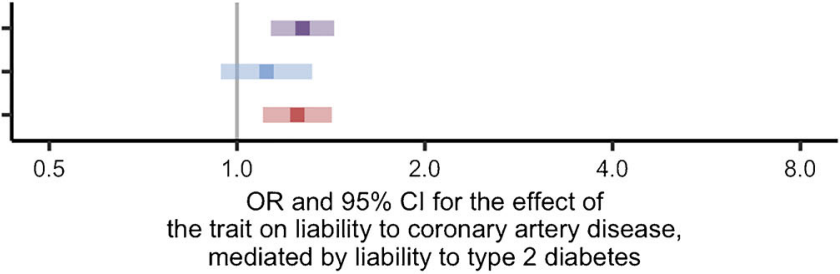

there could be no true mediating effect, so our findings reflect reality. Second, we may lack power to detect a mediating effect as the power requirements for multivariable Mendelian randomisation are greater than univariate approaches and the number of traits considered in this study comes with a high multiple testing burden. Alternatively, the phenotypic complexity of liability to type 2 diabetes may be obscuring effects if, for example, a trait acts on a certain component of liability to type 2 diabetes that does not have a causal effect on liability to atherosclerotic CVD. Partial mediation was observed for two traits: fasting insulin, which is difficult to separate from the clinical definition of type 2 diabetes, and hip circumference, though this particular trait was only an exception for the outcome liability to coronary artery disease. Several of the traits tested, including BMI and waist/hip ratio, were identified as both causes and consequences of liability to type 2 diabetes and so were not studied using two-step Mendelian randomisation for mediation, even if the magnitude of the effects heavily favoured a direction. Despite this, the strong causal effects observed for these traits 
Fig. 3 Two-step Mendelian randomisation for mediation estimates for the total, indirect (mediated by liability to type 2 diabetes) and direct (independent of liability to type 2 diabetes) effects of the indicated risk factors on liability to peripheral artery disease

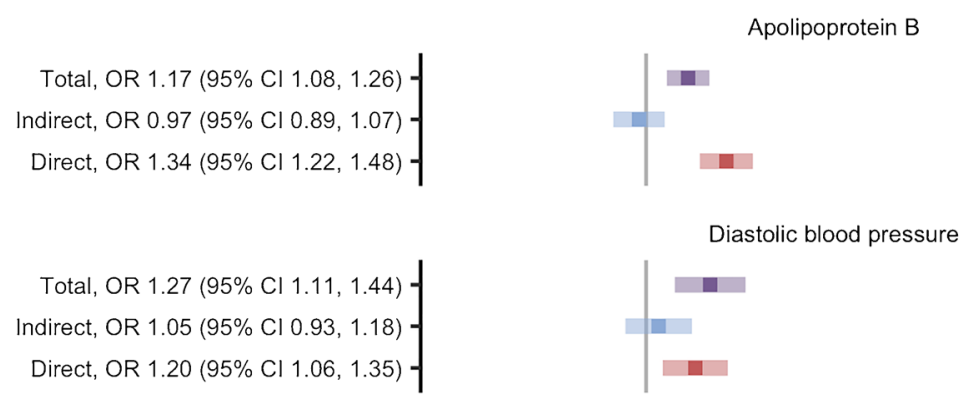

Total, OR $1.56(95 \% \mathrm{Cl} 1.18,2.05)$
Indirect, OR $1.36(95 \% \mathrm{Cl} 0.93,2.00)-$
Direct, OR $1.14(95 \% \mathrm{Cl} 0.96,1.36)$
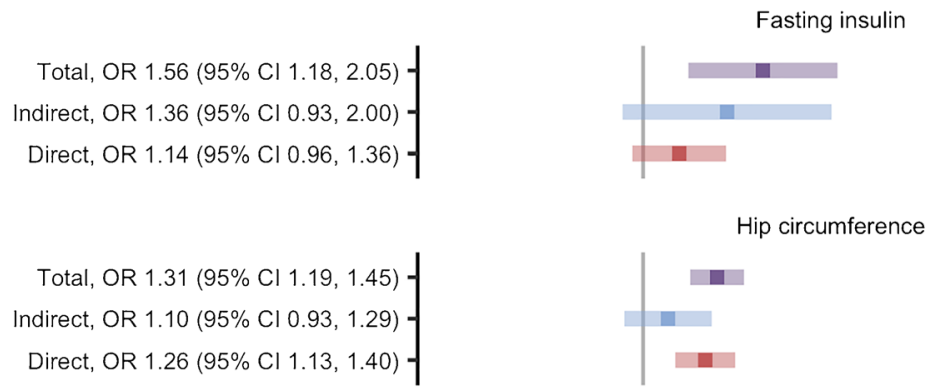

Total, OR $1.15(95 \% \mathrm{Cl} 1.05,1.26)-1$
Indirect, OR $0.97(95 \% \mathrm{Cl} 0.88,1.08)-1$
Direct, OR $1.19(95 \% \mathrm{Cl} 1.11,1.28)-1$

Total cholesterol

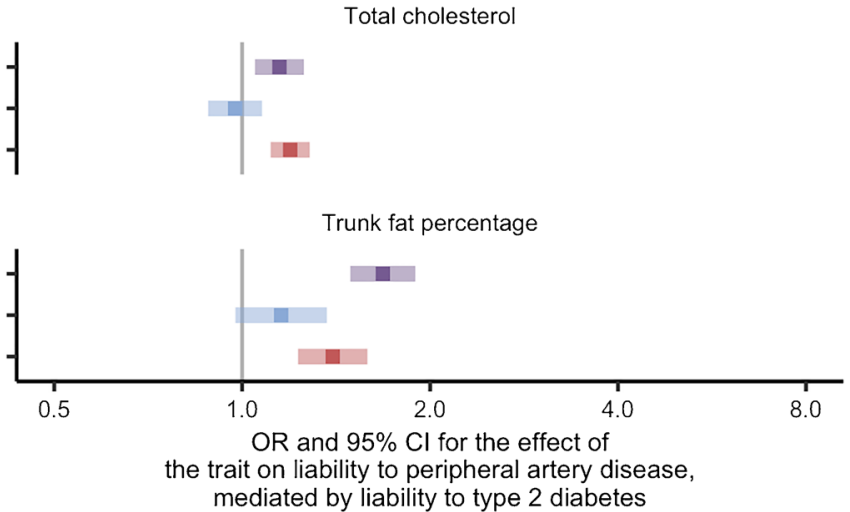

on liability to coronary and peripheral artery disease, without consideration of liability to type 2 diabetes, indicate that they remain important traits for reducing the risk of atherosclerotic CVD outcomes.

Four traits included in this study may be considered as part of the clinical definition of type 2 diabetes or 'endophenotypes' of type 2 diabetes, namely fasting glucose, fasting insulin, glucose and $\mathrm{HbA}_{1 \mathrm{c}}$. Except for fasting insulin, which was found to be a cause but not a consequence of liability to type 2 diabetes, these traits were deemed to have bidirectional relationships with liability to type 2 diabetes when interpreted using the arbitrary 5\% FDR threshold selected for this study. Given the interrelated nature of these glycaemic traits with liability to type 2 diabetes, this is unsurprising and highlights the difficultly in disentangling these effects. Nonetheless, it was important to include these traits in our analysis given our aim of systematically assessing the effects of traits on liability to atherosclerotic CVD risk.

Biologically, our results highlight the centrality of glycaemic traits in the shared causal pathways between type
2 diabetes and CVD, and in mediating the effect of type 2 diabetes on atherosclerotic CVD. There is evidence in the literature to suggest that glycaemic traits may have direct effects on atherosclerotic CVD that are independent of liability to type 2 diabetes. For instance, insulin sensitivity has been shown to be a marker of coronary artery disease risk in nondiabetic populations [39, 40]. Similarly, human genetic evidence suggests that average blood glucose levels linearly influence CHD risk even within the physiologically normal range [41]. Although focus on glycaemic control has variable effects on CVD outcomes among large cohorts of individuals with diabetes, in sum, these findings suggest that glycaemic traits play a key role in the development of atherosclerotic CVD [42, 43].

From a clinical standpoint, our results help clarify the goals of risk factor modification for the prevention of type 2 diabetes, as well as coronary and peripheral artery disease in the setting of type 2 diabetes. They suggest that although diabetes and atherosclerotic CVD share several risk factors, the effects of these traits on atherosclerotic CVD are independent. 
Adiponectin

Albumin
Alcoholic drinks per week

Alkaline phosphatase

Basophil percentage ${ }^{a}$

Body fat

C-reactive protein

Creatinine (enzymatic) in urine

Daytime dozing/sleeping (narcolepsy) ${ }^{a}$

Direct bilirubin

Eosinophil percentage

Getting up in morning

Haematocrit percentage

Haemoglobin concentration

Heart rate

Heel BMD T-score ${ }^{a}$

Lymphocyte percentage

Mean corpuscular haemoglobin concentration

Mean corpuscular volume

Mean platelet (thrombocyte) volume

Mean reticulocyte volume ${ }^{a}$ time to correctly identify matches

Moan time to correctly identify matches

Nap during day ${ }^{a}$

Neuroticism
Neutrophil count

Neutrophil percentage

Platelet count

Platelet crit

Platelet distribution width

Pulse rate

Red blood cell (erythrocyte) distribution width

Sleep duration

Sleeplessness/insomnia

Total bilirubin

Urea $^{\mathrm{a}}$

Urinary sodium/potassium ratio ${ }^{\mathrm{a}}$

Vitamin $D^{\mathrm{a}}$
White blood cell (leucocyte) count

aindicates evidence to support the trait as a consequence of liability to type 2 diabetes

Fig. 4 Venn diagram summarising the traits with evidence to support them as causes of liability to type 2 diabetes, coronary artery disease and peripheral artery disease. ${ }^{a}$ Evidence to support the trait as a

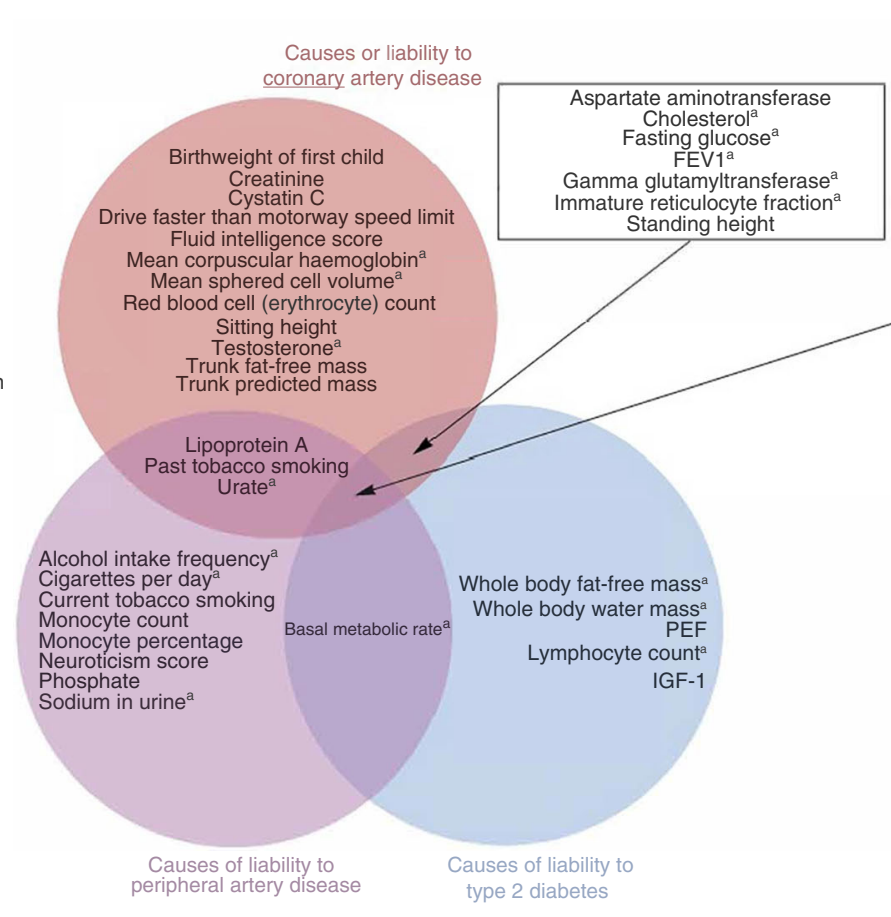

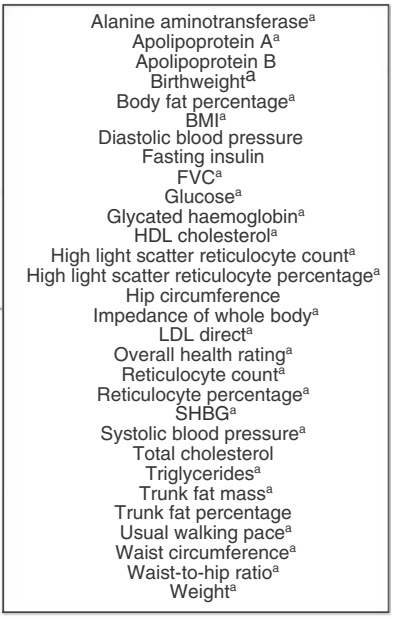

consequence of liability to type 2 diabetes. BMD, bone mineral density; FEV1, forced expiratory volume in $1 \mathrm{~s}$; FVC, forced vital capacity; PEF, peak expiratory flow; SHBG: sex hormone binding globulin
Clinically, these findings argue for broad risk factor modification, rather than targeting glycaemic control as the sole mediator of atherosclerotic CVD risk in individuals with type 2 diabetes. These findings fit within a broader clinical framework built on lifestyle modification, whereby dietary and exercise interventions may influence several cardiovascular risk factors simultaneously, including diabetes/glycaemic traits, obesity, BP and dyslipidaemia.

Our study has some limitations. Mendelian randomisation requires several assumptions to hold for valid estimates to be obtained and two-step Mendelian randomisation for mediation further requires no interaction between the exposure and mediator [6, 7]. Except for relevance, these assumptions cannot be tested. However, where possible, we have performed sensitivity analyses and falsification tests. In addition, our study may be subject to weak instrument bias as a small number of instruments have $F$ statistics that fall below the common (arbitrary) threshold of 10 . We report these results, with their $F$ statistics, but encourage readers to be cautious in the inferences made from these estimates. Our study may also be biased due to the non-collapsibility of ORs, which can impact estimates as a result of summary statistics from logistic regression being used for binary mediators (such as liability to type 2 diabetes) and outcomes (such as liability to coronary and peripheral artery disease) [9]. We assessed this possibility by repeating our analyses with summary statistics from a novel GWAS that used a linear model for liability to type 2 diabetes and found little difference in the Mendelian randomisation estimates we obtained (ESM Methods 2; ESM Fig. 6). This indicates that non-collapsibility of ORs is unlikely to have impacted our results. In addition, our study may be affected by horizontal pleiotropy. We used MR-Egger estimators to investigate whether our results were sensitive to assumptions about the structure of pleiotropy and found some evidence that a small number of traits may have horizontally pleiotropic effects. Finally, our study was restricted to individuals of European or mixed ancestry due to the broad range of GWAS required for the analysis. Consequently, the generalisability of the findings from this study is limited to comparable European or mixed ancestry populations.

In conclusion, we have used a Mendelian randomisation framework to separate the effects of continuous traits from liability to type 2 diabetes and aid our understanding of their relationships with liability to coronary and peripheral artery disease. Our analysis suggests that some key traits, including diastolic BP and hip circumference, act independently of liability to type 2 diabetes. These traits are therefore important for understanding atherosclerotic CVD risk regardless of an individual's liability to type 2 diabetes.

Supplementary Information The online version of this article (https://doi. org/10.1007/s00125-022-05653-1) contains peer-reviewed but unedited supplementary material.

Acknowledgements Quality control filtering of the UK Biobank data was conducted by R. Mitchell, G. Hemani, T. Dudding, Corbin, S. 
Harrison and L. Paternoster (Bristol Medical School, University of Bristol) as described in the published protocol [44]. The MRC IEU UK Biobank GWAS pipeline was developed by B. Elsworth, R. Mitchell, C. Raistrick, L. Paternoster, G. Hemani and T. Gaunt (Bristol Medical School, University of Bristol) [45]. The authors thank Million Veteran Program (MVP) staff, researchers, and volunteers, who have contributed to MVP, and especially participants who previously served their country in the military and now generously agreed to enrol in the study (see https://www.research.va.gov/mvp/ for more details). The citation for MVP is [22]. This research is based on data from the Million Veteran Program, Office of Research and Development, Veterans Health Administration, and was supported by the Veterans Administration (VA) Cooperative Studies Program (CSP) award \#no. G002.

Data availability All data used in this study are publicly available. We accessed genome-wide association study summary statistics for the traits from the IEU OpenGWAS database (https://gwas.mrcieu.ac.uk/), for liability to type 2 diabetes from the DIAMANTE consortium (https:// www.diagram-consortium.org/), for liability to coronary artery disease from the CARDIoGRAM consortium (http://www.cardiogramplusc4d. org/) and for liability to peripheral artery disease from dbGAP (https:// www.ncbi.nlm.nih.gov/gap/). Approved dbGAP access to phs001672. v6.p1 was provided to BFV (dbGAP project ID: 27398).

Funding This research was supported by the U.S. Department of Veterans Affairs (IK2-CX001780 to SMD). This publication does not represent the views of the Department of Veterans Affairs or the United States Government. VMW, ARC, NMD, GDS and TRG are members of the Medical Research Council Integrative Epidemiology Unit, which is supported by the Medical Research Council and the University of Bristol (MC_UU_00011/1, MC_UU_00011/4 and MC_UU_00011/6). VMW is also supported by the COVID-19 Longitudinal Health and Wellbeing National Core Study, which is funded by the Medical Research Council (MC_PC_20059). ARC is also supported by the University of Bristol British Heart Foundation Accelerator Award (AA/18/7/34219). NMD is also supported by a Norwegian Research Council (grant no. 295989). MSU is supported by NIDDK K23DK114551. MGL is supported by the Institute for Translational Medicine and Therapeutics of the Perelman School of Medicine at the University of Pennsylvania and the NIH/NHLBI National Research Service Award postdoctoral fellowship (T32HL007843). BFV is supported by the NIH/NIDDK (DK126194 and DK101478).

Authors' relationships and activities TRG receives funding from Biogen for research unrelated to this manuscript. SMD receives research support from RenalytixAI and personal consulting fees from Calico Labs, outside the scope of the current research. All other authors declare that there are no relationships or activities that might bias, or be perceived to bias, their work.

Contribution statement VMW, BFV, TRG and SMD contributed to the design of the work. VMW performed the data analysis and drafted the article. All authors contributed to the data interpretation, critical revision of the article, and final approval for publication. VMW is the guarantor of this work.

Open Access This article is licensed under a Creative Commons Attribution 4.0 International License, which permits use, sharing, adaptation, distribution and reproduction in any medium or format, as long as you give appropriate credit to the original author(s) and the source, provide a link to the Creative Commons licence, and indicate if changes were made. The images or other third party material in this article are included in the article's Creative Commons licence, unless indicated otherwise in a credit line to the material. If material is not included in the article's Creative Commons licence and your intended use is not permitted by statutory regulation or exceeds the permitted use, you will need to obtain permission directly from the copyright holder. To view a copy of this licence, visit http://creativecommons.org/licenses/by/4.0/.

\section{References}

1. Bellou V, Belbasis L, Tzoulaki I, Evangelou E (2018) Risk factors for type 2 diabetes mellitus: An exposure-wide umbrella review of meta-analyses. PLoS One 13(3):e0194127. https://doi.org/10.1371/ journal.pone. 0194127

2. Malakar AK, Choudhury D, Halder B, Paul P, Uddin A, Chakraborty S (2019) A review on coronary artery disease, its risk factors, and therapeutics. J Cell Physiol 234(10):16812-16823. https://doi.org/10.1002/jcp. 28350

3. Song P, Rudan D, Zhu Y et al (2019) Global, regional, and national prevalence and risk factors for peripheral artery disease in 2015: an updated systematic review and analysis. Lancet Glob Health 7(8): e1020-e1030. https://doi.org/10.1016/S2214-109X(19)30255-4

4. Ahmad OS, Morris JA, Mujammami M et al (2015) A Mendelian randomization study of the effect of type-2 diabetes on coronary heart disease. Nat Commun 6:7060. https://doi.org/10.1038/ ncomms 8060

5. Criqui MH, Aboyans V (2015) Epidemiology of Peripheral Artery Disease. Circ Res 116(9):1509-1526. https://doi.org/10.1161/ CIRCRESAHA. 116.303849

6. Davey Smith G, Ebrahim S (2003) 'Mendelian randomization': can genetic epidemiology contribute to understanding environmental determinants of disease? Int J Epidemiol 32(1):1-22. https://doi. org/10.1093/ije/dyg070

7. Davey Smith G, Hemani G (2014) Mendelian randomization: genetic anchors for causal inference in epidemiological studies. Hum Mol Genet 23(R1):R89-R98. https://doi.org/10.1093/hmg/ ddu328

8. Relton CL, Davey Smith G (2012) Two-step epigenetic Mendelian randomization: a strategy for establishing the causal role of epigenetic processes in pathways to disease. Int J Epidemiol 41(1):161176. https://doi.org/10.1093/ije/dyr233

9. Carter AR, Sanderson E, Hammerton G et al (2021) Mendelian randomisation for mediation analysis: current methods and challenges for implementation. Eur J Epidemiol 36(5):465-478. https://doi.org/10.1007/s10654-021-00757-1

10. Burgess S, Davies NM, Thompson SG (2016) Bias due to participant overlap in two-sample Mendelian randomization. Genet Epidemiol 40(7):597-608. https://doi.org/10.1002/gepi.21998

11. Ference BA, Yoo W, Alesh I et al (2012) Effect of Long-Term Exposure to Lower Low-Density Lipoprotein Cholesterol Beginning Early in Life on the Risk of Coronary Heart DiseaseA Mendelian Randomization Analysis. J Am Coll Cardiol 60(25): 2631-2639. https://doi.org/10.1016/j.jacc.2012.09.017

12. Nelson CP, Hamby SE, Saleheen D et al (2015) Genetically Determined Height and Coronary Artery Disease. N Engl J Med 372(17):1608-1618. https://doi.org/10.1056/NEJMoa1404881

13. Corbin LJ, Richmond RC, Wade KH et al (2016) BMI as a Modifiable Risk Factor for Type 2 Diabetes: Refining and Understanding Causal Estimates Using Mendelian Randomization. Diabetes 65(10):3002-3007. https://doi.org/10. 2337/db16-0418

14. Larsson SC, Bäck M, Rees JMB, Mason AM, Burgess S (2020) Body mass index and body composition in relation to 14 cardiovascular conditions in UK Biobank: a Mendelian randomization study. Eur Heart J 41(2):221-226. https://doi.org/10.1093/eurheartj/ ehz388 
15. Huang Y, Xu M, Xie L et al (2016) Obesity and peripheral arterial disease: A Mendelian Randomization analysis. Atherosclerosis 247: 218-224. https://doi.org/10.1016/j.atherosclerosis.2015.12.034

16. Gill D, Zuber V, Dawson J et al (2021) Risk factors mediating the effect of body mass index and waist-to-hip ratio on cardiovascular outcomes: Mendelian randomization analysis. Int J Obes 45(7): 1428-1438. https://doi.org/10.1038/s41366-021-00807-4

17. Elsworth B, Lyon M, Alexander T et al (2020) The MRC IEU OpenGWAS data infrastructure. bioRxiv 2020.08.10.244293. https://doi.org/10.1101/2020.08.10.244293

18. Sudlow C, Gallacher J, Allen N et al (2015) UK Biobank: An Open Access Resource for Identifying the Causes of a Wide Range of Complex Diseases of Middle and Old Age. PLoS Med 12(3): e1001779. https://doi.org/10.1371/journal.pmed.1001779

19. Mahajan A, Taliun D, Thurner M et al (2018) Fine-mapping type 2 diabetes loci to single-variant resolution using high-density imputation and islet-specific epigenome maps. Nat Genet 50(11):15051513. https://doi.org/10.1038/s41588-018-0241-6

20. Nikpay M, Goel A, Won H-H et al (2015) A comprehensive 1,000 Genomes-based genome-wide association meta-analysis of coronary artery disease. Nat Genet 47(10):1121-1130. https://doi.org/ 10.1038/ng.3396

21. Klarin D, Lynch J, Aragam K et al (2019) Genome-wide association study of peripheral artery disease in the Million Veteran Program. Nat Med 25(8):1274-1279. https://doi.org/10.1038/ s41591-019-0492-5

22. Gaziano JM, Concato J, Brophy M et al (2016) Million Veteran Program: A mega-biobank to study genetic influences on health and disease. J Clin Epidemiol 70:214-223. https://doi.org/10.1016/j. jclinepi.2015.09.016

23. Bowden J, Davey Smith G, Burgess S (2015) Mendelian randomization with invalid instruments: effect estimation and bias detection through Egger regression. Int J Epidemiol 44(2):512-525. https://doi.org/10.1093/ije/dyv080

24. Spiller W, Davies NM, Palmer TM (2019) Software application profile: mrrobust - a tool for performing two-sample summary Mendelian randomization analyses. Int J Epidemiol 48(3):684690. https://doi.org/10.1093/ije/dyy195

25. Hemani G, Zheng J, Elsworth B et al (2018) The MR-Base platform supports systematic causal inference across the human phenome. eLife 7:e34408. https://doi.org/10.7554/eLife.34408

26. Benjamini Y, Hochberg Y (1995) Controlling the False Discovery Rate: A Practical and Powerful Approach to Multiple Testing. J R Stat Soc Ser B Methodol 57(1):289-300

27. Sanderson E, Davey Smith G, Windmeijer F, Bowden J (2019) An examination of multivariable Mendelian randomization in the single-sample and two-sample summary data settings. Int J Epidemiol 48(3):713-727. https://doi.org/10.1093/ije/dyy262

28. Churchhouse C, Neale B (2017) Rapid GWAS of thousands of phenotypes for 337,000 samples in the UK Biobank. Available from http://www.nealelab.is/blog/2017/7/19/rapid-gwas-ofthousands-of-phenotypes-for-337000-samples-in-the-uk-biobank. Accessed 9 Dec 2021

29. Dastani Z, Hivert M-F, Timpson N et al (2012) Novel loci for adiponectin levels and their influence on type 2 diabetes and metabolic traits: a multi-ethnic meta-analysis of 45,891 individuals. PLoS Genet 8(3):e1002607. https://doi.org/10.1371/journal.pgen. 1002607

30. Liu M, Jiang Y, Wedow R et al (2019) Association studies of up to 1.2 million individuals yield new insights into the genetic etiology of tobacco and alcohol use. Nat Genet 51(2):237-244. https://doi. org/10.1038/s41588-018-0307-5

31. Lu Y, Day FR, Gustafsson S et al (2016) New loci for body fat percentage reveal link between adiposity and cardiometabolic disease risk. Nat Commun 7:10495. https://doi.org/10.1038/ ncomms 10495

32. Yengo L, Sidorenko J, Kemper KE et al (2018) Meta-analysis of genome-wide association studies for height and body mass index in 700000 individuals of European ancestry. Hum Mol Genet 27(20):3641-3649. https://doi.org/10.1093/hmg/ddy271

33. Scott RA, Lagou V, Welch RP et al (2012) Large-scale association analyses identify new loci influencing glycemic traits and provide insight into the underlying biological pathways. Nat Genet 44(9): 991-1005. https://doi.org/10.1038/ng.2385

34. den Hoed M, Eijgelsheim M, Esko T et al (2013) Identification of heart rate-associated loci and their effects on cardiac conduction and rhythm disorders. Nat Genet 45(6):621-631. https://doi.org/10. 1038/ng.2610

35. Okbay A, Baselmans BML, Neve J-ED et al (2016) Genetic variants associated with subjective well-being, depressive symptoms, and neuroticism identified through genome-wide analyses. Nat Genet 48(6):624-633. https://doi.org/10.1038/ng.3552

36. Willer CJ, Schmidt EM, Sengupta S et al (2013) Discovery and refinement of loci associated with lipid levels. Nat Genet 45(11): 1274-1283. https://doi.org/10.1038/ng.2797

37. Zanetti D, Bergman H, Burgess S, Assimes TL, Bhalla V, Ingelsson E (2020) Urinary Albumin, Sodium, and Potassium and Cardiovascular Outcomes in the UK Biobank: Observational and Mendelian Randomization Analyses. Hypertension 75(3):714-722. https://doi. org/10.1161/HYPERTENSIONAHA.119.14028

38. Shungin D, Winkler TW, Croteau-Chonka DC et al (2015) New genetic loci link adipose and insulin biology to body fat distribution. Nature 518(7538):187-196. https://doi.org/10.1038/ nature14132

39. Reaven G (2012) Insulin Resistance and Coronary Heart Disease in Nondiabetic Individuals. Arterioscler Thromb Vasc Biol 32(8): 1754-1759. https://doi.org/10.1161/ATVBAHA.111.241885

40. Festa A, D'Agostino R, Hanley AJG, Karter AJ, Saad MF, Haffner SM (2004) Differences in Insulin Resistance in Nondiabetic Subjects With Isolated Impaired Glucose Tolerance or Isolated Impaired Fasting Glucose. Diabetes 53(6):1549-1555. https://doi. org/10.2337/diabetes.53.6.1549

41. Burgess S, Malik R, Liu B et al (2021) Dose-response relationship between genetically proxied average blood glucose levels and incident coronary heart disease in individuals without diabetes mellitus. Diabetologia 64(4):845-849. https://doi.org/10.1007/s00125-02005377-0

42. The Action to Control Cardiovascular Risk in Diabetes Study Group (2008) Effects of intensive glucose lowering in type 2 diabetes. N Engl J Med 358(24):2545-2559. https://doi.org/10.1056/ NEJMoa0802743

43. Nathan DM, for the DCCT/EDIC Research Group (2014) The Diabetes Control and Complications Trial/Epidemiology of Diabetes Interventions and Complications Study at 30 Years: Overview. Diabetes Care 37(1):9-16. https://doi.org/10.2337/ dc13-2112

44. Mitchell R, Hemani G, Dudding T, Corbin L, Harrison S, Paternoster L (2019) UK Biobank Genetic Data: MRC-IEU Quality Control, version 2. Available from https://data.bris.ac.uk/ data/dataset/1ovaau5sxunp2cv8rcy88688v. Accessed 9 Dec 2021

45. Mitchell R, Elsworth BL, Raistrick CA, Paternoster L, Hemani G, Gaunt TR (2019) MRC IEU UK Biobank GWAS pipeline version 2. Available from https://data.bris.ac.uk/data/dataset/ pnoat8cxo0u52p6ynfaekeigi. Accessed 9 Dec 2021

Publisher's note Springer Nature remains neutral with regard to jurisdictional claims in published maps and institutional affiliations. 